\title{
MODELAGEM DA EXTRAÇÃO DE ÓLEOS ESSENCIAIS EMPREGANDO COEFICIENTE DE DIFUSÃO VARIÁVEL
}

Doi:http://dx.doi.org/10.1590/1809-4430-Eng.Agric.v35n2p 302-312/2015

\section{JENIFFER C. SILVEIRA ${ }^{1}$, ANDRÉA O. S. DA COSTA ${ }^{2}$, ESLY F. DA COSTA JUNIOR ${ }^{3}$}

RESUMO: Óleos essenciais são compostos químicos voláteis, característicos por sua fragrância e frequentes atividades antimicrobianas e antioxidantes. São extraídos dos tricomas de plantas aromáticas a partir de diversos métodos diferentes de extração. As indústrias dão preferência à extração por arraste a vapor (destilação a vapor), por ser um processo tradicional, de simples operação e baixo custo. A modelagem matemática deste processo é um passo inevitável para projetar plantas industriais de extração de óleo essencial visando a boas condições operacionais. $\mathrm{O}$ modelo empregado neste trabalho é baseado na difusão do óleo no interior da folha. Apresentam-se o procedimento de discretização deste modelo por método de diferenças finitas e a validação deste por comparação com a solução analítica. Dados da literatura de perfis de rendimento por tempo de extração foram empregados para a estimação do coeficiente de difusão. Propôs-se a melhoria do modelo por meio da estimação de parâmetros de equações empíricas para a descrição do coeficiente de difusão como função da concentração de óleo. A partir desta modificação, o modelo não possui mais solução analítica, o que justifica o procedimento numérico adotado. $\mathrm{O}$ modelo foi validado atra vés de um conjunto de dados disponíveis na literatura.

PALAVRAS-CHAVE: óleos essenciais, modelagem, estimação de parâmetros.

\section{MODELING OF ESSENTIAL OIL EXTRACTION WITH VARIABLE DIFFUSION COEFFICIENT}

\begin{abstract}
Essential oils are volatile chemical compounds, characteristic for its fragrance and frequent antimicrobial and antioxidant activities. Essential oils are extracted from the glandular trichomes of herbs from various extraction methods. Industries give preference to extraction by steam distillation, for its traditional method, simple operation and low cost. The mathematical modeling of this process is an inevitable step for designing industrial plants of essential oil extraction with good operating condition. The model used in this article is based on the diffusion of the oil within the leaf. The discretization procedure of this model is presented through finite differences and its validation by comparing with the analytical solution. Data of profiles yield by extraction time were used to estimate the diffusion coefficient. In order to improve the model empirical equations were applied to describe de diffusion coefficient as a function of the oil concentration and the parameters of these equations were estimated. Using this modification, the model has no longer analytical solution, which justifies the adopted numerical procedure. The model was validated using data from literature.
\end{abstract}

KEYWORDS : essential oils, mathematical modeling, parameter estimation.

\section{INTRODUÇÃO}

Provenientes do metabolismo celular das plantas, os óleos essenciais são compostos químicos voláteis encontrados em regiões denominadas tricomas. São característicos por sua fragrância, aparência oleosa e facilidade para volatilizar (FILIPPIS, 2001). Devido às frequentes atividades

\footnotetext{
${ }^{1}$ Graduanda em Eng Química, Departamento de Engenharia Rural, UFES - Universidade Federal do Espírito Santo, Campus de Alegre, Alto Universitário, s/n, Caixa Postal 16, Guararema/Alegre - ES, Fone: (28) 981116-4241, jeniffercristinasilveira@hotmail.com

${ }^{2} \mathrm{Eng}^{\circ}$ Químico, Prof. Doutor, Departamento de Engenharia Rural, UFES - Universidade Federal do Espírito Santo, Campus de Alegre, Alto Universitário, s/n, Caixa Postal 16, Guararema /Alegre-ES, andreaosc@y ahoo.com.br

${ }^{3}$ Eng $^{\circ}$ Químico, Prof. Doutor, Departamento de Engenharia Rural, UFES - Universidade Federal do Espírito Santo, Campus de Alegre, Alto Universitário, s/n, Caixa Postal 16, Guararema /Alegre - ES, esly @ pq.cnpq.br 
biológica, antimicrobiana e antioxidante (NAVARRETE et al., 2011), óleos essenciais despertam interesse em diversas indústrias, tais como alimentícia, farmacêutica, cosméticos, perfumaria, higiene e limpeza (SILVEIRA et al., 2012 a). Eles são também empregados como matéria-prima na fabricação de tintas e na agricultura, para controle bioló gico (BIASI \& DESCHAMPS, 2009).

Para isolar tais óleos, são empregados diferentes métodos de extração. Entretanto, a composição do óleo pode variar significativamente, dependendo do método a ser utilizado (CASSEL et al., 2009). Alguns dos métodos de extração mais utilizados são: hidrodestilação, destilação a vapor e extração por fluido supercrítico (YUSOFF et al.,2011).

A destilação por arraste a vapor é utilizada principalmente para materiais sensíveis à temperatura (CERPA et al.,2008), sendo baseada na diferença de volatilidade de determinados compostos presentes na matéria-prima. As indústrias dão preferência a este método de extração, pois, devido à sua maior simplicidade e economia, permite tratar de uma única vez quantidades significativas de material vegetal (STEFFANI, 2003).

A modelagem matemática de destilação a vapor é um passo inevitável para projetar plantas industriais de extração de óleo essencial, visando a boas condições operacionais (CASSEL et al., 2009).

A maneira pela qual um modelo matemático é desenvolvido permite sua classificação como fenomenológico (teórico), empírico ou híbrido (PINTO \& LAGE, 2001). Quanto à resolução matemática, os modelos podem ser denominados como lineares (parâmetros dispostos linearmente) ou não lineares (THOLON \& QUEIROZ, 2009). Outra classificação útil refere-se à possibilidade de as propriedades serem uniformes no espaço (modelo a parâmetros concentrados) ou não uniformes no espaço (modelo a parâmetros distribuídos) (PINTO \& LAGE, 2001).

O objetivo deste trabalho consiste na modelagem da extração de óleos essenciais utilizando um modelo a parâmetros distribuídos na fase sólida. Propõe-se a implementação do modelo, para a descrição do processo de extração de óleos essenciais, considerando inicialmente o coeficiente de difusão constante. Assim como o trabalho desenvolvido por SIQUEIRA et al. (2012), que estuda o resfriamento de morangos com ar forçado, e o estudo desenvolvido por SILVA et al. (2012), que trata da transferência de massa durante o processo de secagem de bananas, o modelo apresentado inicialmente possui solução analítica. Entretanto, nestes dois trabalhos da literatura e no presente trabalho, a inserção de modificações no modelo faz com que ele não tenha mais solução analítica e só possa ser resolvido numericamente. Neste trabalho, a modificação proposta consiste numa equação empírica para a descrição do coeficiente de difusão como função da concentração de óleo na folha. $\mathrm{O}$ modelo resultante sem solução analítica é resolvido pela discretização da direção espacial por diferenças finitas. Em seguida, os parâmetros do modelo serão estimados a partir dos dados experimentais disponíveis na literatura.

\section{MATERIAL E MÉTODOS}

O modelo considerado como base para o desenvolvimento deste trabalho baseia-se na difusão na fase sólida (folha da planta na qual se extrai o óleo essencial). Sendo assim, considera-se o perfil de concentração dentro da fase sólida e, portanto, o modelo é a parâmetros distribuídos. Esta estraté gia foi empregada em CASSEL \& VARGAS (2006) e CASSEL et al. (2009).

Para o desenvolvimento do modelo, CASSEL \& VARGAS (2006) e CASSEL et al. (2009) assumem as seguintes considerações: a etapa controladora do processo de transferência de massa do óleo das folhas para o vapor é a difusão; a distribuição do óleo essencial em todas as folhas é homogênea no início do processo de extração; considera-se na fase sólida somente a variação da concentração ao longo da espessura da folha da planta, tendo-se um problema unidimensional em coordenadas cartesianas; a lei da Fick da difusão modela o processo difusivo; após o início da extração, não há resistência à transferência de massa da superfície da planta para o vapor, e a concentração de óleo na fase vapor é muito baixa e considerada nula. 
A partir das considerações realizadas, tem-se o equacionamento composto pelo balanço de massa na fase sólida dado por:

$$
\begin{aligned}
& \frac{\partial^{2} \mathrm{C}_{\mathrm{a}}(\mathrm{x}, \mathrm{t})}{\partial \mathrm{x}^{2}}=\frac{1}{\mathrm{D}} \frac{\partial \mathrm{C}_{\mathrm{a}}(\mathrm{x}, \mathrm{t})}{\partial \mathrm{t}} \\
& \text { para } \mathrm{t}=0, \mathrm{C}_{\mathrm{a}}(\mathrm{x}, 0)=\mathrm{C}_{\mathrm{a} 0} \\
& \text { para } \mathrm{x}=0, \mathrm{C}_{\mathrm{a}}(0, \mathrm{t})=0 \\
& \text { para } \mathrm{x}=\mathrm{L}, \mathrm{C}_{\mathrm{a}}(\mathrm{L}, \mathrm{t})=0
\end{aligned}
$$

em que,

$\mathrm{C}_{\mathrm{a}}(\mathrm{x}, \mathrm{t})$ é a concentração de óleo na fase sólida;

D é o coeficiente de difusão do óleo na fase sólida;

$\mathrm{C}_{\mathrm{a} 0}$ é a concentração inicial de óleo essencial na planta aromática, e

Lé a espessura da planta.

Dado que os modelos de CASSEL \& VARGAS (2006) e CASSEL et al. (2009) assumem que o coeficiente de difusão é constante, o modelo apresentado possui solução analítica. Segundo estes trabalhos, esta solução é dada pela [eq. (5)].

$$
\left.C_{a}(x, t)=\sum_{n=1}^{\infty} \frac{4 C_{a 0}}{n \pi} e^{\left[-D\left(\frac{n \pi}{L}\right)^{2} t\right.}\right] \operatorname{sen}\left(\frac{n \pi}{L} x\right)
$$

SILVEIRA et al. (2012 b) verificaram, a partir da implementação da [eq. (5)], que ocorreu um equívoco na apresentação da solução analítica do modelo de CASSEL \& VARGAS (2006) e CASSEL et al. (2009). A equação foi corrigida, utilizando-se somente os termos ímpares da série, conforme BOYCE \& DIPRIMA (2010), tornando-se:

$$
C_{a}(x, t)=\sum_{m=0}^{\infty} \frac{4 C_{a 0}}{(2 m+1) \pi} e^{\left(\frac{-(2 m+1)^{2} \pi^{2} D}{L^{2}} t\right)} \operatorname{sen}\left(\frac{(2 m+1) \pi}{L} x\right)
$$

Para calcular a taxa de massa extraída, SILVEIRA et al. (2012 b) desenvolveram a [eq. (7)], obtida a partir do produto da área superficial pelo fluxo mássico no contorno.

$$
\dot{\mathrm{m}}(\mathrm{t})=\frac{4 \mathrm{C}_{\mathrm{ag}} \mathrm{DA}}{\mathrm{L}} \sum_{\mathrm{m}=0}^{\infty} \mathrm{e}^{-\mathrm{D}\left(\frac{(2 \mathrm{~m}+1) \pi}{\mathrm{L}}\right)^{2}} \mathrm{t}
$$

em que,

m(t) é a taxa de massa extraída como função do tempo e A é a área superficial.

De acordo com CASSEL \& VARGAS (2006) e CASSEL et al. (2009), a massa extraída como função do tempo é dada pela integração da [eq. (7)] e é apresentada na [eq. (8)].

$$
\mathrm{m}(\mathrm{t})=\frac{8 \mathrm{~m}_{\mathrm{g} 0}}{\pi^{2}} \sum_{\mathrm{m}=0}^{\infty} \frac{1-\mathrm{e}^{-\frac{(2 \mathrm{~m}+1)^{2} \mathrm{\pi}^{2} \mathrm{Dt}}{\mathrm{L}^{2}}}}{(2 \mathrm{~m}+1)^{2}}
$$

em que, 
$\mathrm{m}(\mathrm{t})$ é a massa extraída como função do tempo, e

$\mathrm{m}_{\mathrm{a} 0}$ é a massa inicial de óleo contida em todas as plantas. Ressalta-se que $\mathrm{m}_{\mathrm{a} 0}$ é o produto do volume (área de um dos contornos $(=\mathrm{A} / 2)$ vezes a espessura, $\mathrm{L}$ ) pela concentração inicial de óleo essencial nas plantas $\left(\mathrm{C}_{\mathrm{a} 0}\right)$.

De acordo com CASSEL \& VARGAS (2006) e CASSEL et al. (2009), o grau de extração de óleo é então definido pela [eq. (9)]:

$$
\mathrm{e}(\mathrm{t})=\frac{\mathrm{m}(\mathrm{t})}{\mathrm{m}(\infty)}=\frac{\sum_{\mathrm{m}=0}^{\infty} \frac{\mathrm{e}^{-\frac{(2 \mathrm{~m}+1)^{2} \pi^{2} \mathrm{Dt}}{\mathrm{L}^{2}}}}{\sum_{\mathrm{m}=0}^{\infty} \frac{1}{(2 \mathrm{~m}+1)^{2}}}}{\frac{1}{(2 \mathrm{~m}+1}}
$$

Conforme já discutido, são propostas neste trabalho modificações no modelo que tornam o mesmo sem solução analítica. Desta forma, a equação que descreve a difusão de óleo essencial no interior da partícula, [eq. (1)], juntamente com suas condições iniciais e de contorno, respectivamente, eqs. (2), (3) e (4), foram discretizadas na coordenada espacial pelo método de diferenças finitas, obtendo-se um sistema de equações diferenciais ordinárias (EDOs). A implementação do algoritmo com as equações discretizadas a serem integradas foi realizada no software MATLAB, sendo o integrador utilizado neste software uma implementação do método de Runge-Kutta de $2^{\mathrm{a}}$ e $3^{\mathrm{a}}$ ordem.

De posse da implementação da solução numérica, o modelo foi utilizado em novas estimações de parâmetros, por meio da solução de um problema de otimização baseado no somatório dos quadrados dos resíduos entre os valores simulados e experimentais. O algoritmo de otimização empregado foi a implementação do método Simplex no MATLAB. Os parâmetros estimados foram o coeficiente de difusão (no caso do mesmo constante) ou os parâmetros das relações empregadas no cálculo deste coeficiente e a concentração inicial de óleo essencial. A estimativa da concentração inicial de óleo essencial foi necessária porque este valor não é reportado em CASSEL \& VARGAS (2006) e CASSEL et al. (2009), já que o mesmo não é necessário para o cômputo do grau de extração, segundo a [eq. (9)].

Aproveitando-se da situação de coeficiente de difusão constante, uma análise da convergência da malha foi realizada em comparação com a solução analítica.

Para comparar o desempenho do modelo implementado na simulação do processo de extração, foram empregados dados experimentais da extração do óleo essencial de citronela (Cymbopogon winterianus) (CASSEL \& VARGAS, 2006). Modificações foram propostas ao modelo, objetivando sua melhoria, considerado um coeficiente de difusão variável dependente da concentração ao longo da espessura da folha, definido pelas seguintes relações: $D=\alpha . C(x, t)$, $D=\alpha \cdot C(x, t)+\beta, D=\alpha / C(x, t)+\beta$ e $D=\alpha \cdot C^{2}(x, t)+\beta$. Após alterações no modelo, os parâmetros $\alpha$ e $\beta$ foram estimados.

Com o objetivo de testar a validade do modelo e das modificações propostas, eles foram aplicados a outro conjunto de dados da literatura (LEAL, 2008) para a extração do óleo essencial de camomila (Chamomilla recutita) em escala laboratorial.

As condições utilizadas na simulação para as duas espécies empregadas são apresentadas conforme a Tabela 1 . 
TABELA 1. Condições utilizadas na simulação. Conditions used in the simulation.

\begin{tabular}{lccc}
\hline & Massa de material $(\mathrm{kg})$ & Espessura da folha $(\mathrm{m})$ Tempo de extração $(\mathrm{s})$ \\
\hline Citronela (Cymbopogon winterianus) & 0,040 & $5,42 \times 10^{-4}$ & 6840 \\
\hline Camomila (Chamomilla recutita) & 0,065 & $5,25 \times 10^{-4}$ & 18000 \\
\hline
\end{tabular}

Na Tabela 2, são apresentados os dados experimentais utilizados na simulação para as diferentes espécies.

TABELA 2. Dados experimentais utilizados na simulação. Experimental data used in the simulation.

\begin{tabular}{cccc}
\hline \multicolumn{2}{c}{ Citronela $($ Cymbopogon winterianus $)$} & \multicolumn{2}{c}{ Camomila $($ Chamomilla recutita $)$} \\
\hline Tempo $(\mathrm{s})$ & Rendimento $\left(\mathrm{kg}_{\text {óleo essencial }} \times \mathrm{kg}_{\text {matérial seco }}\right)$ & Tempo $(\mathrm{s})$ & Rendimento $\left(\mathrm{kg}_{\text {óleo essencial }} \times \mathrm{kg}_{\text {matérial seco }}\right)$ \\
\hline 0 & 0 & 0 & 0 \\
180 & 0,00213 & 278 & 0,019305 \\
360 & 0,00425 & 598 & 0,032432 \\
540 & 0,00850 & 875 & 0,045560 \\
720 & 0,01063 & 1196 & 0,057143 \\
990 & 0,01275 & 1473 & 0,068726 \\
1260 & 0,01488 & 1793 & 0,081853 \\
1530 & 0,01700 & 2071 & 0,090347 \\
2160 & 0,01913 & 2391 & 0,098069 \\
3960 & 0,02125 & 2669 & 0,107336 \\
5400 & 0,02338 & 2989 & 0,116602 \\
6840 & 0,02550 & 3267 & 0,124324 \\
- & - & 3587 & 0,131274 \\
- & - & 4761 & 0,154440 \\
- & - & 5979 & 0,176834 \\
- & - & 7174 & 0,195367 \\
- & - & 8370 & 0,213900 \\
- & - & 9566 & 0,229344 \\
- & - & 10782 & 0,240154 \\
- & - & 11979 & 0,254054 \\
- & - & 13174 & 0,264865 \\
- & - & 14349 & 0,274903 \\
- & - & 15544 & 0,285714 \\
- & - & 16740 & 0,294981 \\
- & - & 17957 & 0,301931 \\
\hline
\end{tabular}

\section{RESULTADOS E DISCUSSÃO}

Visando à resolução numérica do problema proposto, a espessura da folha do material vegetal foi dividida em $n$ intervalos iguais, partindo da região central da folha (aproveitando-se da simetria).

Por meio do método de diferenças finitas, aplicado à coordenada espacial, as eqs. (1), (2) e (4), respectivamente, balanço de massa e suas condições iniciais e de contorno, foram discretizadas obtendo-se as eqs. (10), (11) e (12). A discretização da condição de simetria resultou na [eq. (13)].

$$
\frac{\mathrm{dC}_{\mathrm{ai}}}{\mathrm{dt}}=\mathrm{D} \frac{\left[\mathrm{C}_{\mathrm{g}(\mathrm{i}+1)}-2 \mathrm{C}_{\mathrm{a}(\mathrm{i})}+\mathrm{C}_{\mathrm{g}(\mathrm{i}-1)}\right]}{\Delta \mathrm{x}^{2}}
$$


para $\mathrm{t}=0: \quad \mathrm{C}_{\mathrm{a}}\left(\mathrm{x}_{\mathrm{i}}, 0\right)=\mathrm{C}_{\mathrm{a} 0} \quad$ onde $\mathrm{i}=1,2,3, \ldots, \mathrm{n}$

$\operatorname{para} \mathrm{x}=\mathrm{L} / 2: \mathrm{C}_{\mathrm{a}}(\mathrm{L} / 2, \mathrm{t})=0$

$\operatorname{para} \mathrm{x}=0: \frac{\mathrm{dC}_{\mathrm{a}}}{\mathrm{dt}}=\frac{\left[\mathrm{C}_{\mathrm{a}(1)}-\mathbf{C}_{\mathrm{a}(0)}\right]}{\Delta \mathrm{x}}=\mathbf{0}, \log 0 \quad \mathrm{C}_{\mathrm{a}(0)}=\mathrm{C}_{\mathrm{a}(1)}$

O sistema de EDOs encontrado, a partir da discretização das equações do modelo, foi resolvido utilizando-se da rotina de integração ode23 do MATLAB.

A partir da análise da convergência da malha, em comparação com a solução analítica, obteve-se o número de pontos discretizados (n) equivalentes a 115. Na Figura 1, são apresentadas curvas para alguns valores de n, considerando-se os perfis de concentração para 3 valores distintos de tempo de extração.

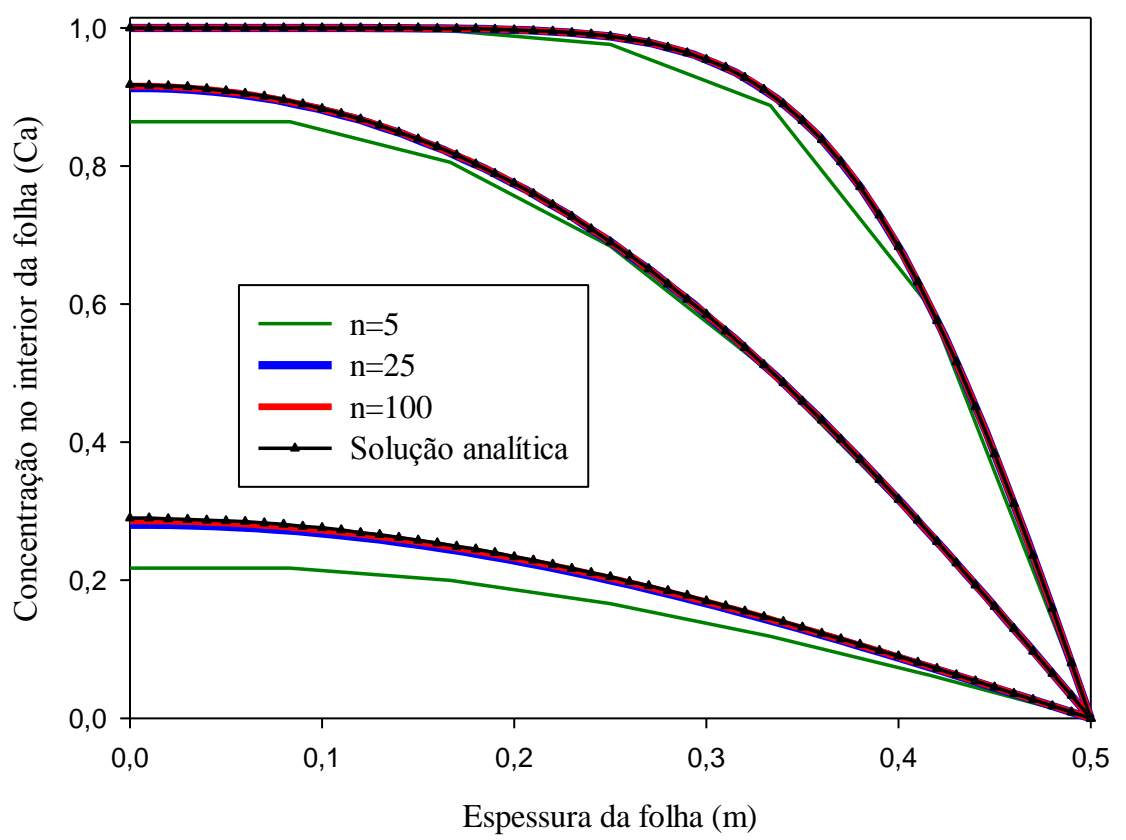

FIGURA 1. Análise da convergência da malha em comparação com a solução analítica. Analysis of convergence of the mesh compared with analytical solution.

A partir dos valores de concentração nos pontos de discretização, o rendimento no instante t, $\mathrm{r}(\mathrm{t})$, pode ser calculado a partir da diferença entre a concentração inicial de óleo essencial na folha e o valor médio de concentração de óleo na folha no instante t, segundo a [eq. (14)].

$$
\mathrm{r}(\mathrm{t})=\mathrm{C}_{\mathrm{a} 0}-\frac{1}{(\mathrm{n}+1)}\left[\frac{\mathrm{C}_{\mathrm{a}(0)}}{2}+\sum_{\mathrm{i}=2}^{\mathrm{n}} \mathrm{C}_{\mathrm{a}}(\mathrm{i})\right]
$$

A partir dos dados disponíveis no trabalho de CASSEL \& VARGAS (2006), reproduziu-se a curva de rendimento por tempo de extração (Figura 2) para os dados simulados em comparação aos dados experimentais. Utilizou-se, para a solução do modelo, do coeficiente de difusão estimado por CASSEL \& VARGAS (2006), sendo este $\mathrm{D}=1,6 \times 10^{-11} \mathrm{~m}^{2} \times \mathrm{s}^{-1}$, e a concentração inicial de óleo

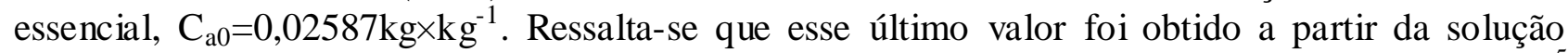
analítica em conjunto com os dados experimentais. Para esta simulação, obtiveram-se $11,78 \times 10^{-5}$ como somatório dos erros ao quadrado (SEQ), quantificado através do desvio entre as curvas experimental e simulada. 
Os parâmetros $\mathrm{D}$ e $\mathrm{C}_{\mathrm{a} 0}$ foram estimados pela resolução do problema de otimização que consiste na minimização de SEQ. Os resultados foram: $\mathrm{D}=1,353 \times 10^{-11} \mathrm{~m}^{2} \times \mathrm{s}^{-1}$ e $\mathrm{C}_{\mathrm{a} 0}=0,0254 \mathrm{~kg}_{\mathrm{N}} \mathrm{kg}^{-1}$, com o $\mathrm{SEQ}=2,31 \times 10^{-5}$. O perfil de rendimento por tempo de extração é apresentado na Figura 2.

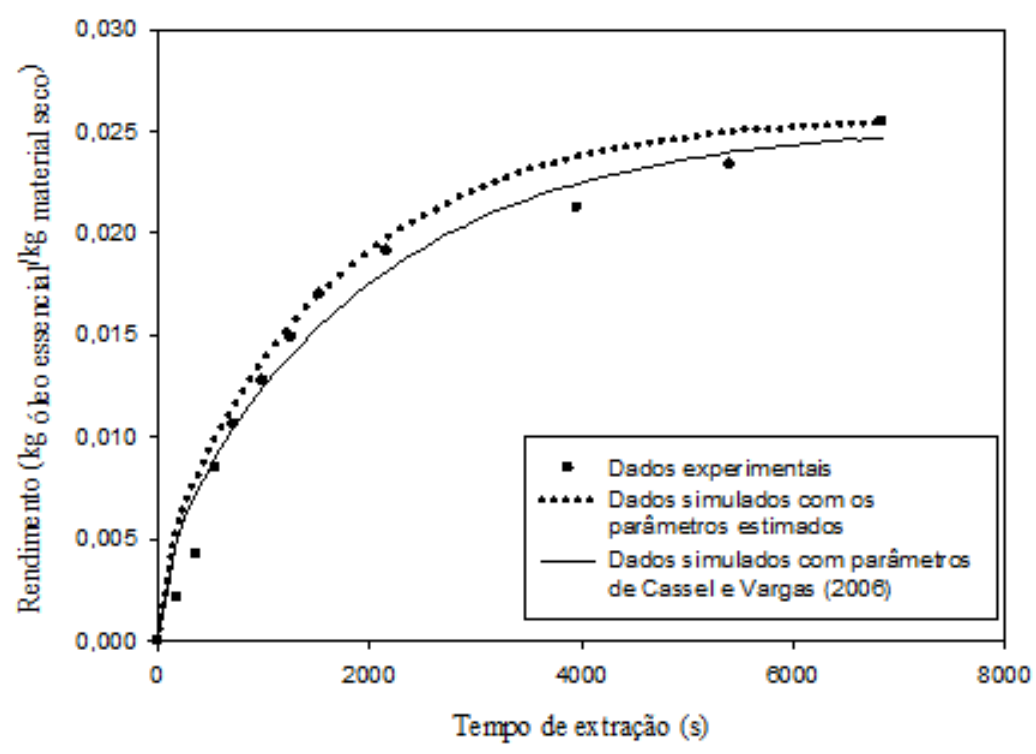

FIGURA 2. Perfil de rendimento por tempo de extração, utilizando-se os parâmetros de CASSEL \& VARGAS (2006) e os parâmetros estimados. Profile yield for time extraction using the parameters shown by CASSEL \& VARGAS (2006) and the estimated parameters.

Analisando-se os perfis de rendimento apresentados pela Figura 2, nota-se, empregando-se os parâmetros estimados neste trabalho, que há uma melhoria no desempenho do modelo. Esta melhoria também pode ser observada comparando-se os valores de SEQ.

Conforme já discutido, foram testadas funções paramétricas para a variação do coeficiente de difusão como função da concentração. Os resultados são apresentados na Figura 3 e na Tabela 3. 

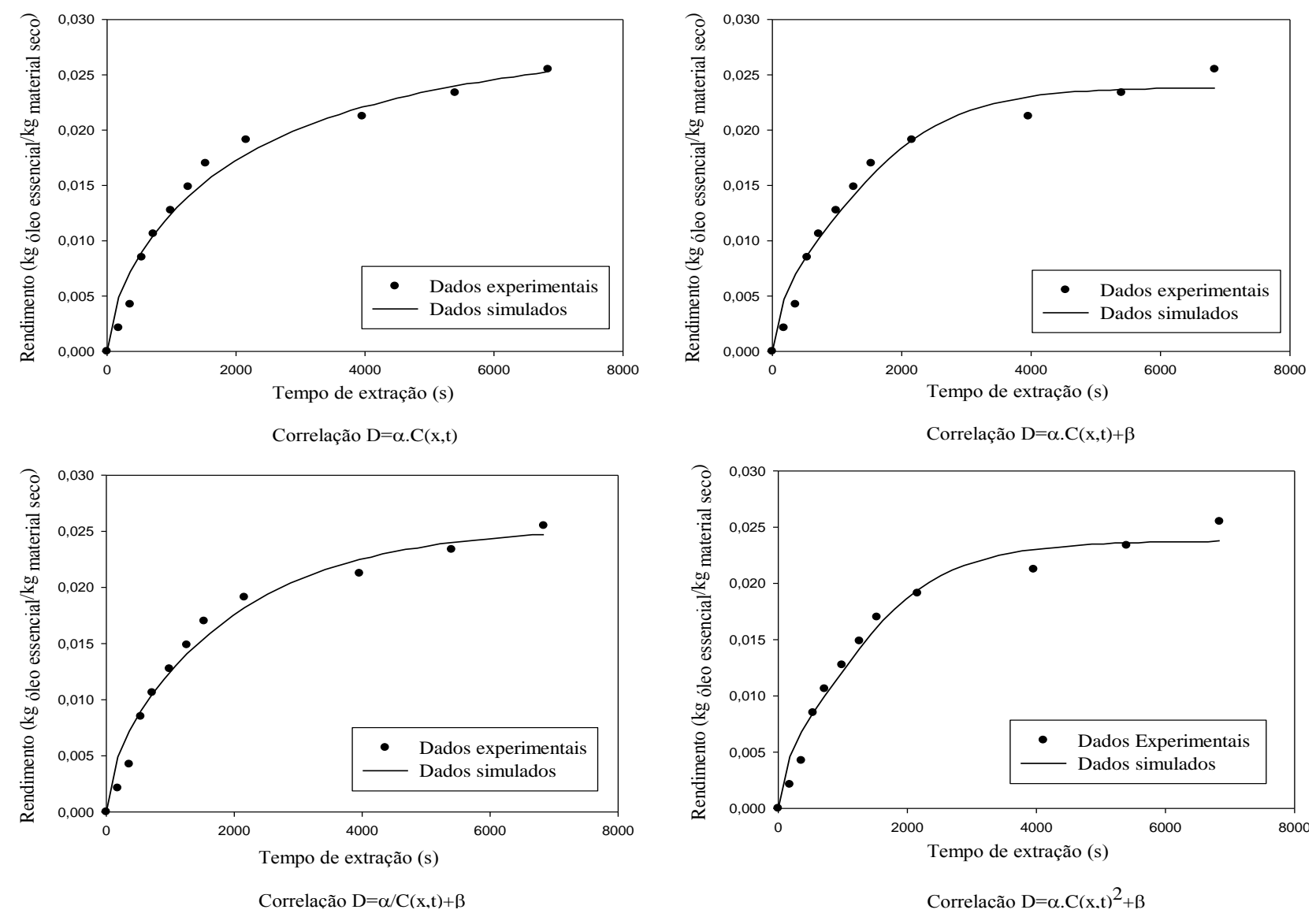

FIGURA 3. Perfil de rendimento por tempo de extração, utilizando-se das correlações propostas. Profile yield for extraction time using the proposed correlations.

TABELA 3. Correlações para o coeficiente de difusão modificado. Correlations for the modified diffusion coefficient.

\begin{tabular}{|c|c|c|}
\hline Correlações & Parâmetros estimados & SEQ \\
\hline $\mathrm{D}=\alpha \cdot \mathrm{C}(\mathrm{x}, \mathrm{t})$ & $\begin{array}{c}\alpha=38,56 \times 10^{-11} \mathrm{~m}^{2} \times \mathrm{kg}_{\text {matérial seco }} \times\left(\mathrm{kg}_{\text {óleo essencial }}\right)^{-1} \times \mathrm{s}^{-1} \\
\mathrm{C}_{\mathrm{a} 0}=0,032 \mathrm{~kg}_{\text {óleo essencial }} \times\left(\mathrm{kg}_{\text {mateŕrial seco }}\right)^{-1}\end{array}$ & $2,29 \times 10^{-5}$ \\
\hline $\mathrm{D}=\alpha \cdot \mathrm{C}(\mathrm{x}, \mathrm{t})+\beta$ & $\begin{array}{c}\alpha=-72,49 \times 10^{-11} \mathrm{~m}^{2} \mathrm{~kg}_{\text {matérial seco }} \times\left(\mathrm{kg}_{\text {óleo essencial }}\right)^{-1} \times \mathrm{s}^{-1} \\
\beta=2,83 \times 10^{-11} \mathrm{~m}^{2} \times \mathrm{s}^{-1} \\
\mathrm{C}_{\mathrm{a} 0}=0,024 \mathrm{~kg}_{\text {óleo essencial }} \times\left(\mathrm{kg}_{\text {matérial seco }}\right)^{-1}\end{array}$ & $2,2610^{-5}$ \\
\hline $\mathrm{D}=\alpha / \mathrm{C}(\mathrm{x}, \mathrm{t})+\beta$ & $\begin{array}{c}\alpha=-1,0 \times 10^{-15} \mathrm{~m}^{2} \times \mathrm{kg}_{\text {matérial seco }} \times\left(\mathrm{kg}_{\text {óleo essencial }}\right)^{-1} \times \mathrm{s}^{-1} \\
\beta=1,35 \times 10^{-11} \mathrm{~m}^{2} \times \mathrm{s}^{-1} \\
\mathrm{C}_{\mathrm{a} 0}=0,025 \mathrm{~kg}_{\text {óleo essencial }} \times\left(\mathrm{kg}_{\text {matérial seco }}\right)^{-1}\end{array}$ & $2,31 \times 10^{-5}$ \\
\hline$D=\alpha \cdot C^{2}(x, t)+\beta$ & $\begin{array}{c}\alpha=-3,13 \times 10^{-8} \mathrm{~m}^{2} \times \mathrm{kg}_{\text {matérial seco }} \times\left(\mathrm{kg}_{\text {óleo essencial }}\right)^{-1} \times \mathrm{s}^{-1} \\
\beta=2,63 \times 10^{-11} \mathrm{~m}^{2} \times \mathrm{s}^{-1} \\
\mathrm{C}_{\mathrm{a} 0}=0,024 \mathrm{~kg}_{\text {óleo essencial }} \times\left(\mathrm{kg}_{\text {matérial seco }}\right)^{-1}\end{array}$ & $2,14 \times 10^{-5}$ \\
\hline
\end{tabular}

Após análise da Figura 4 em conjunto com os dados disponíveis na Tabela 1, verificou-se que ocorreu uma diminuição no valor do SEQ, exceto na terceira correlação proposta, na qual SEQ é praticamente idêntico àquele obtido para o coeficiente de difusão constante $\left(\mathrm{SEQ}=2,31 \times 10^{-5}\right)$.

Com a quarta correlação, foi possível obter o menor SEQ, dentre as correlações testadas, sendo este equivalente a $2,14 \times 10^{-5}$.

A partir dos dados apresentados em LEAL (2008), os parâmetros do modelo para o coeficiente de difusão constante foram estimados, e os seguintes valores foram encontrados,

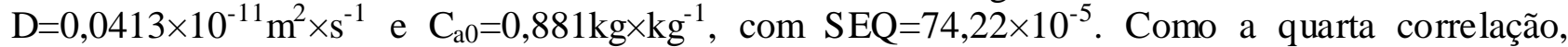
$\mathrm{D}=\alpha \cdot \mathrm{C}^{2}(\mathrm{x}, \mathrm{t})+\beta$, apresentou os melhores resultados para $\mathrm{SEQ}$, seus parâmetros também foram estimados para os dados de LEAL (2008), sendo obtidos: $\alpha=0,0497 \times 10^{-11} \mathrm{~m}^{2} \times \mathrm{s}^{-1}, \beta=-0,0016 \times 10^{-}$ 
${ }^{11} \mathrm{~m}^{2} \times \mathrm{s}^{-1}$ e $\mathrm{C}_{\mathrm{a} 0}=1,10{\mathrm{~kg} \times \mathrm{kg}^{-1}}$, com $\mathrm{SEQ}=69,44 \times 10^{-5}$. Os perfis de rendimento por tempo de extração para os dados de LEAL (2008) são apresentados na Figura 4.

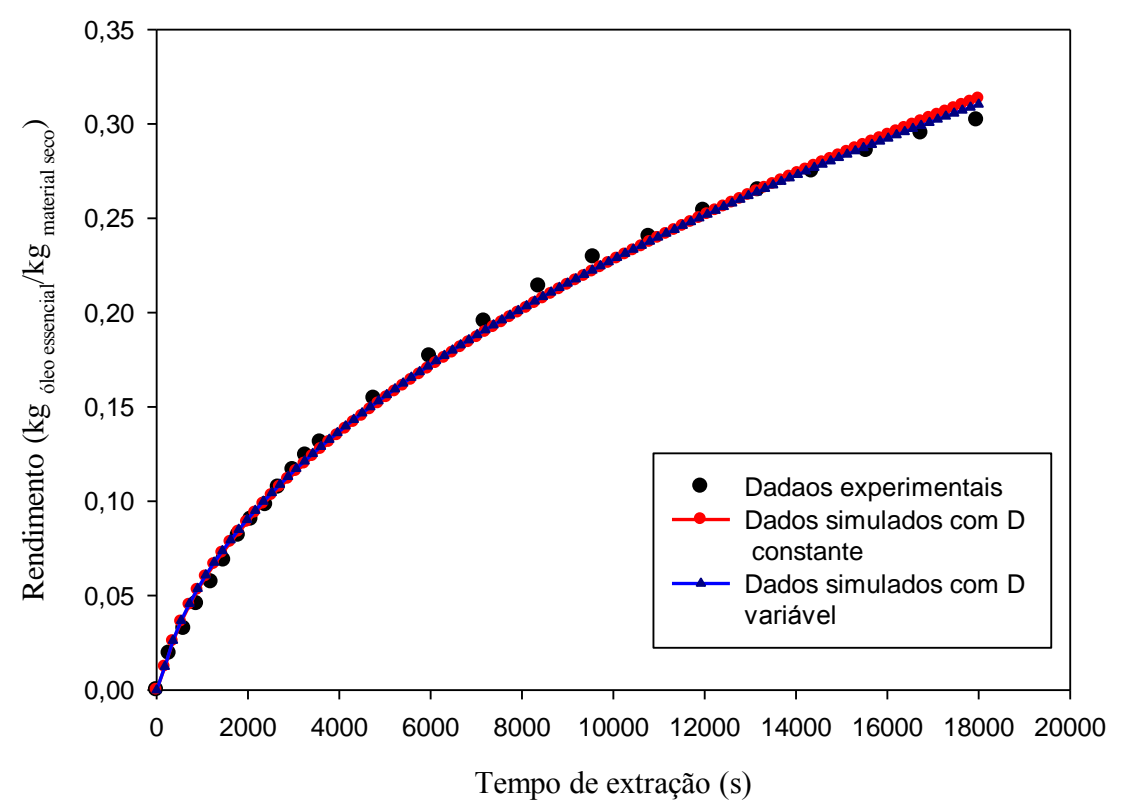

FIGURA 4. Perfis de rendimento por tempo de extração para os dados apresentados em LEAL (2008). Profile yields by extraction time for the data presented in LEAL (2008).

Analisando a Figura 4 em conjunto com os valores obtidos para o SEQ, observou-se que, durante o início do processo até o tempo próximo a $14.000 \mathrm{~s}$, o comportamento das curvas simuladas foi semelhante. No entanto, a partir do tempo de $14.000 \mathrm{~s}$ até o final do processo, observou-se maior adequação da curva simulada com D variável em comparação aos dados experimentais. Mais além, o valor mais elevado de SEQ para os dados de LEAL (2008), quando comparado aos dados de CASSEL \& VARGAS (2006), deve-se exclusivamente aos maiores valores de rendimento da extração para a camomila, quando comparada com a citronela.

\section{CONCLUSÕES}

No presente trabalho, são propostas e testadas modificações no modelo a parâmetro distribuído para a simulação da extração de óleo essencial proposto por CASSEL \& VARGAS (2006) e CASSEL et al. (2009). A partir das modificações propostas, o modelo não possui solução analítica e apresenta-se um procedimento para a discretização do mesmo pelo método de diferenças finitas.

As modificações testadas baseiam-se em equações paramétricas para o cômputo do coeficiente de difusão como função da concentração de óleo. Utilizando-se dos dados apresentados em CASSEL \& VARGAS (2006), estes parâmetros foram estimados pela minimização do somatório dos resíduos ao quadrado. Observou-se que uma relação quadrática para o coeficiente de difusão apresenta melhor desempenho na descrição dos dados experimentais.

Por meio da simulação de um novo conjunto de dados apresentado em LEAL (2008), verificou-se novamente a adequação das modificações propostas na simulação das condições experimentais da extração de óleo essencial.

\section{AGRADECIMENTOS}

À FAPES (Fundação de Amparo à Pesquisa do Espírito Santo), pela concessão de bolsas de iniciação científica e de pesquisador capixaba. 
Ao CNPq (Conselho Nacional de Desenvolvimento Científico e Tecnológico), pela concessão de bolsa de produtividade em desenvolvimento tecnoló gico e extensão inovadora.

\section{REFERÊNCIAS}

BIASI, L.A.; DESCHAMPS, C. Plantas aromáticas: do cultivo à produção de óleo essencial. Curitiba: Layer Studio Gráfico e Editora, 2009.

BOYCE, W. E.; DIPRIMA, R. C. Equações diferenciais elementares e problemas de valores de contorno. 9. ed. Rio de Janeiro: LTC, 2010.

CASSEL, E.; VARGAS, R. M. F. Experiments and Modeling of the Cymbopogon winterianus Essential Oil Extraction by Steam Distillation. Journal of the Mexican Chemical Society, Mexico, v. 50, p. 126-129, 2006.

CASSEL, E.; VARGAS, R. M. F.; MARTINEZ, N.; LORENZO, D.; DELLACASSA, E. Steam distillation modeling for essential oil extraction process. Industrial Crops and Products, Amsterdam, v. 29, p.171-176, 2009.

CERPA, M. G. et al. Modelling steam distillation of essential oils: aplication to lavandin super oil. AIChE Journal, New York, v. 54, n. 4, p. 909-917, 2008. doi 10.1002/aic.11438.

FILIPPIS, F. DE M. Extração com $\mathrm{CO}_{2}$ supercrítico de óleos essencial de Hon-sho e Ho-shoexperimentos e modelagem. 2001. 114f. Dissertação (Mestrado em Engenharia Química) Departamento de Engenharia Química, Universidade Federal do Rio Grande do Sul, Porto Alegre, 2001.

LEAL, P. F. Estudo comparativo entre os custos de manufatura e as propriedades funcionais de óleos voláteis obtidos por extração supercrítica e destilação por arraste a vapor. 2008. 275f. Tese (Doutorado em Engenharia de Alimentos) - Departamento de Engenharia de Alimentos, Universidade Estadual de Campinas, Campinas, 2008.

NAVARRETE, A.; WALLRAF, S.; MATO, R. B.; COCERO, M. J. Improvement of Essential Oil Steam Distillation by Microwave Pretreatment. I\&EC Research, Washington, v. 50, p. 4667-4671, 2011.

PINTO, J. C.; LAGE, P. L. C. Métodos matemáticos em problemas de engenharia química - Série Escola Piloto em Engenharia Química: COPPE/UFRJ: Rio de Janeiro: Editora E - papers, 2001.

SILVA, C. M. D. P. da S.; SILVA, W. P.; FARIAS, V. S. de O. e GOMES, J. P. Effective diffusivity and convective mass transfer coefficient during the drying of bananas, Engenharia Agrícola, Jaboticabal, v. 32, n.2, p. 342-353, 2012.

SILVEIRA, J. C.; BUSATO, N. V.; COSTA, A. O. S.; COSTA JR., E. F. Levantamento e analise de métodos de extração de óleos essenciais. Enciclopédia Biosfera, Goiânia, v. 8, n. 15, p. 20382052, 2012a.

SILVEIRA, J. C.; BUSATO, N. V.; COSTA, A. O. S.; COSTA JR., E. F. Proposta de modelagem da extração de óleos essenciais utilizando modelo a parâmetros distribuídos na fase solida. In: ENCONTRO LATINO AMERICANO DE INICIACAO CIENTIFICA, 16., 2012, São Jose dos Campos - SP. Anais... São Jose dos Campos: Universidade do Vale do Paraíba, 2012b.

SIQUEIRA, A. J. H.; COSTA, A. O. S.; COSTA Jr., E. F. Modeling and Simulation of Forced-air Cooling of Strawberries Using Variable Convective Coefficient, Engenharia Agricola, Jaboticabal, v. 32, n. 1, p.164-173, 2012.

STEFFANI, E. Modelagem matemática do processo de extração supercrítica de óleo essencial de Ho-Sho (Cinnamomum camphora Nees \& Eberm var. Linaloolifera Fujita) utilizando $\mathrm{CO}_{2} .2003$. 106f. Tese (Doutorado em Engenharia Química)- Departamento de Engenharia Química e 
Engenharia de Alimentos, Centro Tecnológico, Universidade Federal de Santa Catarina, Santa Catarina, 2003.

THOLON, P.; QUEIROZ, S. A. Modelos matemáticos utilizados para descrever curvas de 22 crescimento em aves aplicados ao melhoramento genético animal. Ciência Rural, Santa Maria, v. 39, n. 7, 23 p. 2261-2269, 2009.

YUSOFF, Z. M.; NORDIN, M. N. N.; RAHIMAN, M. H. F.; ADNAN, R.; TAIB, M. N. Characterization of down-flowing steam distillation system using step test analysis. IEEE CSGRC, New York, p. 197-201, 2011. 